\title{
Object Detection In Image Using Predator-Prey Optimization
}

\author{
Ankit Sharma ${ }^{1}$ and Nirbhowjap Singh ${ }^{2}$ \\ ${ }^{1}$ Electronics \& Instrumentation Engineering Department, SVITS, Indore, India \\ ankit.sharma2012@gmail.com \\ ${ }^{2}$ Electronics \& Instrumentation Engineering Department, Thapar University, Patiala, India \\ nirbhowegmail. com
}

\begin{abstract}
Image matching is a key component in almost any image analysis process. Image matching is crucial to a wide range of applications, such as in navigation, guidance, automatic surveillance, robot vision, and in mapping sciences. Any automated system for three-dimensional point positioning must include a potent procedure for image matching. Most biological vision systems have the talent to cope with changing world. Computer vision systems have developed in the same way. For a computer vision system, the ability to cope with moving and changing objects, changing illumination, and changing viewpoints is essential to perform several tasks. Object detection is necessary for surveillance applications, for guidance of autonomous vehicles, for efficient video compression, for smart tracking of moving objects, for automatic target recognition (ATR) systems and for many other applications. Cross-correlation and related techniques have dominated the field since the early fifties. Conventional template matching algorithm based on cross-correlation requires complex calculation and large time for object detection, which makes difficult to use them in real time applications. The shortcomings of this class of image matching methods have caused a slow-down in the development of operational automated correlation systems. In the proposed work particle swarm optimization \& its variants based algorithm is used for detection of object in image. Implementation of this algorithm reduces the time required for object detection than conventional template matching algorithm. Algorithm can detect object in less number of iteration \& hence less time \& energy than the complexity of conventional template matching. This feature makes the method capable for real time implementation. In this paper a description of particle Swarm optimization algorithm is given \& then formulation of the algorithm for object detection using PSO \& its variants is implemented for validating its effectiveness.
\end{abstract}

Keywords- Cross Correlation Co-efficient, Particle swarm optimization, Predator-prey optimization, Pattern Recognition, Template Matching.

\section{INTRODUCTION}

As humans, it is easy (even for a child) to detect the position of the letters, objects, numbers, etc. However, making a computer solve these types of problems in fast manner is a very challenging task. Object detection is a fundamental component of artificial intelligence and computer vision. Object detection methods are used in various areas such as science, engineering, medical applications, etc. Interest in pattern recognition is fast growing in order to deal with the prohibitive amount of information, we encounter in our daily life. Automation is desperately needed to handle this information explosion. Object detection is necessary for surveillance applications, for guidance of autonomous vehicles, for efficient video compression, for smart tracking of moving 
Signal \& Image Processing : An International Journal(SIPIJ) Vol.2, No.1, March 2011

objects, for automatic target recognition (ATR) systems and for many other applications. In the last decades the computer's ability to perform huge amount of calculations, and handle information flows we never thought possible ten years ago has emerged. Despite this a computer can only extract little information from the image in comparison to human being. The way the human brain filters out useful information is not fully known and this skill has not been merged into computer vision science. The aim of this work is to implement a system in MATLAB that is able to faster detection of object in an image. The system should use both fast and advanced algorithms aiming to achieve the exact position of the object in image. The goal is to develop a system with the potential to be implemented in a real time environment. Therefore the system needs to be very fast.

\section{LITERATURE REVIEW}

In [4] a new feature based correspondence algorithm for image matching was proposed. In [5] a procedure for digital image correlation is described which is based on least squares window matching. In [6] the method for fast normalized cross-correlation is proposed. In [7] the problem of crafting visual routines for detection tasks is address. In [8] a generic matching algorithm suitable for many applications where feature extraction is difficult or inaccurate is presented. In [14] a new method for locating object based on valley field is proposed. In [15] problem of locating an object in an image when size and rotation are unknown are examined. In [16] the method of image matching by normalized cross-correlation is proposed. In [17] a novel method which is robust and efficient in extracting objects using Wavelets and Neural Networks is suggested. In [18] the generalized projection function (GPF) is defined. In [19] a novel approach to pattern matching is proposed in which time complexity is reduced by two orders of magnitude compared to traditional approaches. In [20] a robust eye detection algorithm for gray intensity images is presented. The idea of their method was to combine the respective advantages of two existing techniques, Feature based method and template based method, and to overcome their shortcomings.

\section{Template Matching \& Cross Correlation Cofficient}

Template matching is a popular method for pattern recognition. It is defined below:

Definition: Let $I$ be an image of dimension $m \times n$ and $T$ be another image of dimension $p \times q$ such that $\mathrm{p}<\mathrm{m}$ and $\mathrm{q}<\mathrm{n}$ then template matching is defined as a search method which finds out the portion in I of size $\mathrm{p} \times \mathrm{q}$ where $\mathrm{T}$ has the maximum cross correlation coefficient with it.

The normalized cross correlation coefficient is defined as:

$$
\lambda(x, y)=\frac{\sum_{s} \sum_{t} \delta_{I(x+s, y+t)} \delta_{T(s, t)}}{\sum_{s} \sum_{t} \delta_{I(x+s, y+t)}^{2} \sum_{s} \sum_{t} \delta_{T(s, t)}^{2}}
$$

Where,

$$
\begin{aligned}
& \delta_{I(x+s, y+t)}=I(x+s, y+t)-I(x, y), \delta_{T(s, t)}=\mathrm{T}(\mathrm{s}, \mathrm{t})-\mathrm{T} \\
& \mathrm{s} €\{1,2,3, \ldots . . \mathrm{p}\}, \text { and } \mathrm{t} €\{1,2,3, \ldots . . \mathrm{q}\} . \\
& \mathrm{x} €\{1,2,3, \ldots \ldots \mathrm{m}-\mathrm{p}+1\}, \mathrm{y} €\{1,2,3, \ldots \ldots . \mathrm{n}-\mathrm{q}+1\}
\end{aligned}
$$


Signal \& Image Processing : An International Journal(SIPIJ) Vol.2, No.1, March 2011

$$
\begin{gathered}
I(x, y)=\frac{1}{p q} \sum_{s} \sum_{t} I(x+s, y+t) \\
T=\frac{1}{p q} \sum_{s} \sum_{t} T(s, t)
\end{gathered}
$$

The value of cross-correlation coefficient $\gamma$ ranges in $[-1,+1]$. A value of +1 indicates that $T$ is completely matched with $\mathrm{I}(\mathrm{x}, \mathrm{y})$ and -1 indicates complete disagreement. For template matching the template, $\mathrm{T}$ slides over I and $\gamma$ is calculated for each coordinate $(\mathrm{x}, \mathrm{y})$. After completing this calculation, the point which exhibits maximum $\gamma$ is referred to as the match point.

\section{Particle SWarm Optimization}

Particle swarm optimization is a stochastic, population-based search and optimization algorithm for problem solving. It is a kind of swarm intelligence that is based on social-psychological principles and provides insights into social behavior, as well as contributing to engineering applications. The particle swarm optimization algorithm was first described in 1995 by James Kennedy and Russell C. Eberhart. The techniques have evolved greatly since then, and the original version of the algorithm is barely used at present. Social influence and social learning enable a person to maintain cognitive consistency. People solve problems by talking with other people about them, and as they interacts their beliefs, attitudes, and behavior changes, the changes could typically be depicted as the individuals moving toward one another in a socio-cognitive space.

The particle swarm simulates a kind of social optimization. A problem is given, and some way to evaluate a proposed solution to it exists in the form of a fitness function. A communication structure or social network is also defined, assigning neighbors for each individual to interact with a population of individuals defined as random guesses as the problem solutions is initialized. These individuals are candidate solutions and are also known as the particles, hence the name particle swarm. An iterative process to improve these candidate solutions is set in motion. The particles iteratively evaluate the fitness of the candidate solutions and remember the location where they had their best fitness value. The individual's best solution is called the particle best or the local best. Each particle makes this information available to their neighbors. They are also able to see where their neighbors have had best fitness value. Movements through the search space are guided by these successes, with the population usually converging, by the end of a trial, on a problem solution better than that of non-swarm approach using the same methods.

The particle swarm optimization (PSO) algorithm is a population-based search algorithm inspired by the social behavior of birds within a flock. The initial intent of the particle swarm concept was to graphically simulate the graceful and unpredictable choreography of a bird flock, the aim of discovering patterns that govern the ability of birds to fly synchronously, and to suddenly change direction with a regrouping in an optimal formation. From this initial objective, the concept evolved into a simple and efficient optimization algorithm. In PSO, individuals, referred to as particles, are "flown" through hyper dimensional search space. Changes to the position of particles within the search space are based on the social-psychological tendency of individuals to emulate the success of other individuals. The changes to a particle within the swarm are therefore influenced by the experience, or knowledge, of its neighbors. The search behavior of a particle is 
Signal \& Image Processing : An International Journal(SIPIJ) Vol.2, No.1, March 2011

thus affected by that of other particles within the swarm therefore PSO is the kind of symbiotic cooperative algorithm. The consequence of modeling this social behavior is that the search process is such that particles stochastically return toward previously successful regions in the search space. The operation of the PSO is based on the neighborhood principle as social network structure.

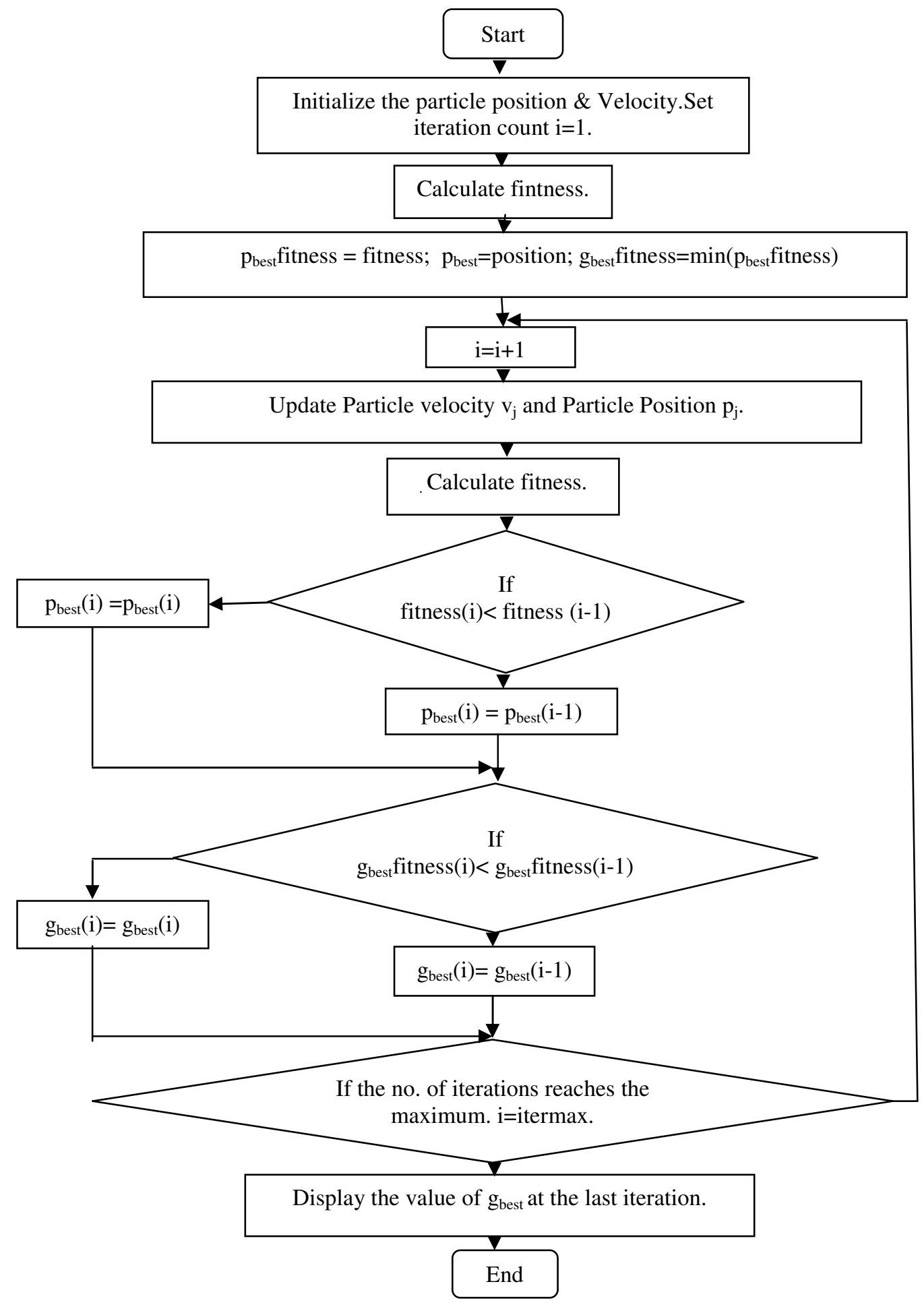




\section{The Predator-Prey OPTIMIZER}

Motivation for developing the predator-prey model was mainly to introduce a mechanism for creating diversity in the swarm at any moment during the run of the algorithm, not depending on the level of convergence already achieved. This allows the "escape" of particles even when convergence of the swarm around a local suboptimum had already occurred. A second, and less practical, motive was to maintain the biological metaphor. The predator-prey model is inspired in the hunt in nature of animals grouped in flocks by one or more predators. When chased, animals have more difficulty to stay around their most preferable places (better pastures, water sources...) and have to search for other locations, free of predators and perhaps even better. This is the effect which is modeled in this algorithm, where the metaphorical better pastures are the function's local sub optima. The predator's objective is to pursue the best individual in the swarm, i.e. the individual that has found the best point in the search space corresponding to the function being optimized. The predator update equations are:

$$
\begin{aligned}
& v_{p}(i)=\mathrm{C}_{4}\left(x_{g}(i-1)-x_{p}(i-1)\right) \\
& x_{p}(i)=x_{p}(i-1)+v_{p}(i)
\end{aligned}
$$

$\mathrm{C}_{4}$ is another random number distributed between 0 and an upper limit and $x_{g}$ is the present position of the best particle in the swarm. The upper limit on $\mathrm{C}_{4}$ allows us to control how fast the predator "catches" the best individual.

The influence of the predator on any individual in the swarm is controlled by a "fear" probability $P_{f}$, which is the probability of a particle changing its velocity in one of the available dimensions due to the presence of the predator.

The dimension where the change will occur is randomly chosen. For some particle $j$, if there is no change in the velocity in a dimension $\mathrm{k}$ the update rules in that dimension still are:

$$
\begin{aligned}
& \mathrm{v}_{\mathrm{jk}}(\mathrm{i})=\mathrm{wv}_{\mathrm{jk}}(\mathrm{i}-1)+\mathrm{C}_{1}\left(\mathrm{p}_{\text {best }(\mathrm{kk})}-\mathrm{x}_{\mathrm{jk}}(\mathrm{i}-1)\right)+\mathrm{C}_{2}\left(\mathrm{~g}_{\text {best }}-\mathrm{x}_{\mathrm{jk}}(\mathrm{i}-1)\right) \\
& \mathrm{x}_{\mathrm{jk}}(\mathrm{i})=\mathrm{x}_{\mathrm{jk}}(\mathrm{i}-1)+\mathrm{v}_{\mathrm{jk}}(\mathrm{i})
\end{aligned}
$$

But if the predator influences the velocity in dimension $\mathrm{k}$, the rule becomes:

$$
\begin{aligned}
& v_{j k}(i)=w v_{j k}(i-1)+C_{1}\left(p_{b e s t(j k)}-x_{j k}(i-1)\right)+C_{2}\left(g_{b e s t}-x_{j k}(i-1)\right)+C_{3} D(d) \\
& x_{j k}(i)=x_{j k}(i-1)+v_{j k}(i)
\end{aligned}
$$

The fourth term in the first equation in (4.5) quantifies the repulsive influence of the predator by modifying the velocity adding a value that is a function of the difference between the position of the predator and the particle. $d$ is the averaged sum of the absolute values of the distances in each dimension. $D(x)$ is an exponential decreasing distance function defined as:

$$
D(x)=a e^{-b x}
$$

$D(x)$ makes the influence of the predator grow exponentially with proximity. The objective of its use is to introduce more perturbation in the swarm when the particles are nearer the predator, 
which usually happens when convergence occurs. When the distance is bigger (e.g. during the initial exploration phase of the swarm, when $w$ is still big), the predator's influence is smaller and usual swarm dynamics take control. The $a$ and $b$ parameter defines the form of the $D$ function: $a$ represents the maximum amplitude of the predator effect over a prey and $b$ permits to control the distance at which the effect is still significant.

The predator effect was designed to take advantage of the use of $w$ as an inertia parameter in the swarm update equations. The idea is to lower the values of $w$, thus forcing a faster convergence, while relying on the predator to maintain population diversity.

\section{EXPERIMENTAL RESULTS \& DisCUSSION}

The algorithms of particle swarm optimization \& Predator-prey approach are applied for solving object detection problem. The performance has been studied for different images and different templates. The results are discussed as -

\section{A.Test Images \& Templates}

Different test images and templates on which the developed algorithms are tested are shown below:



Image (a)

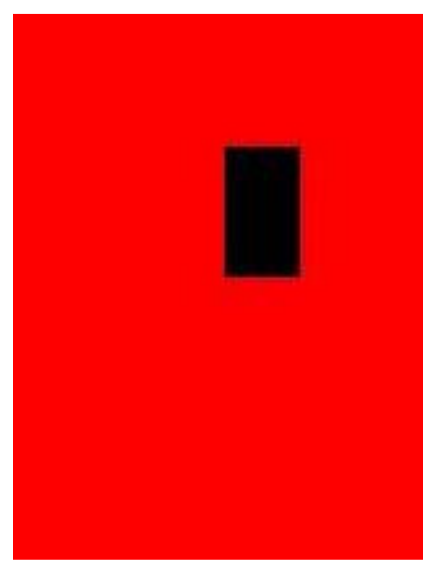

Image (b)

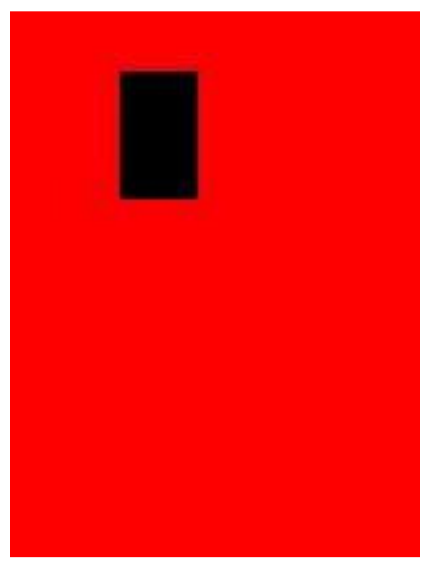

Image (c)

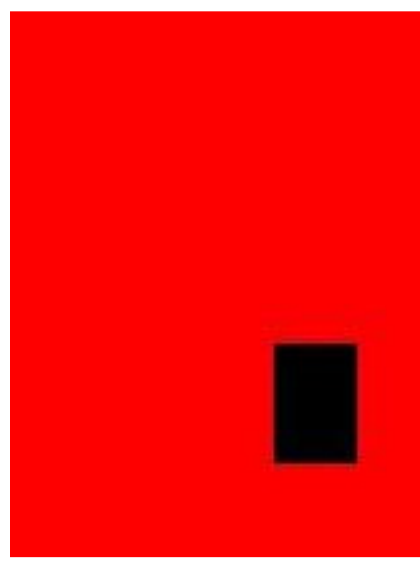

Image (d)



Image (e) 
Signal \& Image Processing : An International Journal(SIPIJ) Vol.2, No.1, March 2011



Image (f)

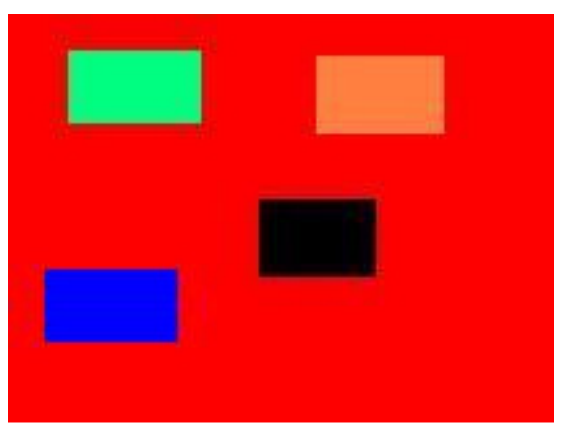

Image (h)

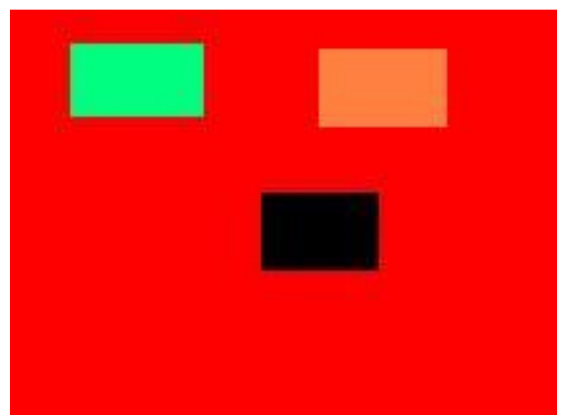

Image (g)

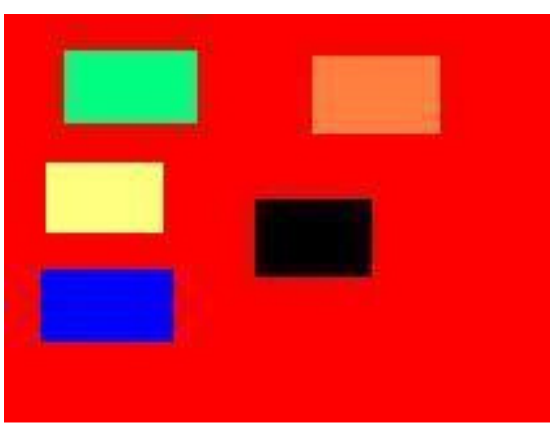

Image (i)

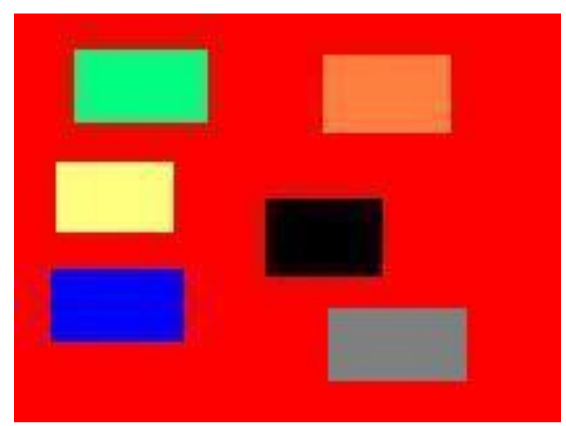

Image (j)

Fig. 1 Different test images 
Signal \& Image Processing : An International Journal(SIPIJ) Vol.2, No.1, March 2011

\section{B.PSO Based Technique}

The proposed PSO based technique was implemented in MATLAB and run under Pentium-4 $(2.79 \mathrm{GHz})$ machine with $512 \mathrm{MB}$ of RAM. Each test image is tested ten times by PSO based object detection program.

\begin{tabular}{|c|c|c|c|c|c|c|c|}
\hline \multirow{2}{*}{ Image } & \multicolumn{6}{|c|}{ PSO Based Algorithm } & \multirow{2}{*}{$\begin{array}{c}\begin{array}{c}\text { Old } \\
\text { Algorithm }\end{array} \\
\text { Time } \\
\text { Taken (In } \\
\text { sec.) }\end{array}$} \\
\hline & $\begin{array}{c}\text { Minimum } \\
\text { Iteration } \\
\text { Taken }\end{array}$ & $\begin{array}{c}\text { Minimum } \\
\text { Time } \\
\text { Taken } \\
\text { (In sec.) }\end{array}$ & $\begin{array}{c}\text { Maximum } \\
\text { Iteration } \\
\text { Taken }\end{array}$ & $\begin{array}{c}\text { Maximum } \\
\text { Time } \\
\text { Taken } \\
\text { (In sec.) }\end{array}$ & $\begin{array}{c}\text { Average } \\
\text { Iteration } \\
\text { Taken }\end{array}$ & $\begin{array}{c}\text { Average } \\
\text { Time } \\
\text { Taken } \\
\text { (In sec.) }\end{array}$ & \\
\hline (a) & 7 & 0.18 & 131 & 2.03 & 38 & 0.60 & 3.85 \\
\hline (b) & 11 & 0.17 & 65 & 1.00 & 32 & 0.50 & 5.56 \\
\hline (c) & 11 & 0.17 & 90 & 1.39 & 33 & 0.5 & 2.87 \\
\hline (d) & 18 & 0.27 & 170 & 2.63 & 51 & 0.81 & 7.33 \\
\hline (e) & 18 & 0.27 & 74 & 1.14 & 48 & 0.70 & 4.19 \\
\hline (f) & 3 & 0.04 & 73 & 1.16 & 32 & 0.50 & 5.70 \\
\hline (g) & 4 & 0.06 & 130 & 2.01 & 38 & 0.60 & 5.70 \\
\hline (h) & 15 & 0.23 & 82 & 1.27 & 45 & 0.70 & 5.70 \\
\hline (i) & 10 & 0.15 & 152 & 2.35 & 56 & 0.8 & 5.70 \\
\hline (j) & 6 & 0.09 & 82 & 1.27 & 44 & 0.60 & 5.70 \\
\hline
\end{tabular}

Table 1 Time consumed and No. of iterations by PSO based algorithm \& Old algorithm

\section{PPO Based Technique}

The proposed PPO based technique was implemented in MATLAB and run under Pentium-4 $(2.79 \mathrm{GHz})$ machine with $512 \mathrm{MB}$ of RAM. Each test image is tested ten times by PPO based object detection program. 
Signal \& Image Processing : An International Journal(SIPIJ) Vol.2, No.1, March 2011

\begin{tabular}{|c|c|c|c|c|c|c|c|}
\hline \multirow{2}{*}{ Image } & \multicolumn{6}{|c|}{ PSO Based Algorithm } & \multirow{2}{*}{$\begin{array}{c}\begin{array}{c}\text { Old } \\
\text { Algorithm }\end{array} \\
\text { Time } \\
\text { Taken (In } \\
\text { sec.) }\end{array}$} \\
\hline & $\begin{array}{c}\text { Minimum } \\
\text { Iteration } \\
\text { Taken }\end{array}$ & $\begin{array}{c}\text { Minimum } \\
\text { Time } \\
\text { Taken } \\
\text { (In sec.) }\end{array}$ & $\begin{array}{c}\text { Maximum } \\
\text { Iteration } \\
\text { Taken }\end{array}$ & $\begin{array}{c}\text { Maximum } \\
\text { Time } \\
\text { Taken } \\
\text { (In sec.) }\end{array}$ & $\begin{array}{c}\text { Average } \\
\text { Iteration } \\
\text { Taken }\end{array}$ & $\begin{array}{c}\text { Average } \\
\text { Time } \\
\text { Taken } \\
\text { (In sec.) }\end{array}$ & \\
\hline (a) & 7 & 0.18 & 131 & 2.03 & 38 & 0.60 & 3.85 \\
\hline (b) & 11 & 0.17 & 65 & 1.00 & 32 & 0.50 & 5.56 \\
\hline (c) & 11 & 0.17 & 90 & 1.39 & 33 & 0.5 & 2.87 \\
\hline (d) & 18 & 0.27 & 170 & 2.63 & 51 & 0.81 & 7.33 \\
\hline (e) & 18 & 0.27 & 74 & 1.14 & 48 & 0.70 & 4.19 \\
\hline (f) & 3 & 0.04 & 73 & 1.16 & 32 & 0.50 & 5.70 \\
\hline (g) & 4 & 0.06 & 130 & 2.01 & 38 & 0.60 & 5.70 \\
\hline (h) & 15 & 0.23 & 82 & 1.27 & 45 & 0.70 & 5.70 \\
\hline (i) & 10 & 0.15 & 152 & 2.35 & 56 & 0.8 & 5.70 \\
\hline (j) & 6 & 0.09 & 82 & 1.27 & 44 & 0.60 & 5.70 \\
\hline
\end{tabular}

Table 1 Time consumed and No. of iterations by PSO based algorithm \& Old algorithm

\section{D.Real time images \& test results with modified algorithm}

PSO \& PPO based algorithms are tested on some real time images, \& it is found that these algorithms are not performing well for finding the position of object in image. Concept of regenerating the population is used for the detection of object in such type of images \& on testing it is found that this algorithm is capable of detecting the position of the object with 10 times reduction in time as compared to conventional template matching algorithms. Different test images $\&$ templates on which program is tested is shown below 
Signal \& Image Processing : An International Journal(SIPIJ) Vol.2, No.1, March 2011
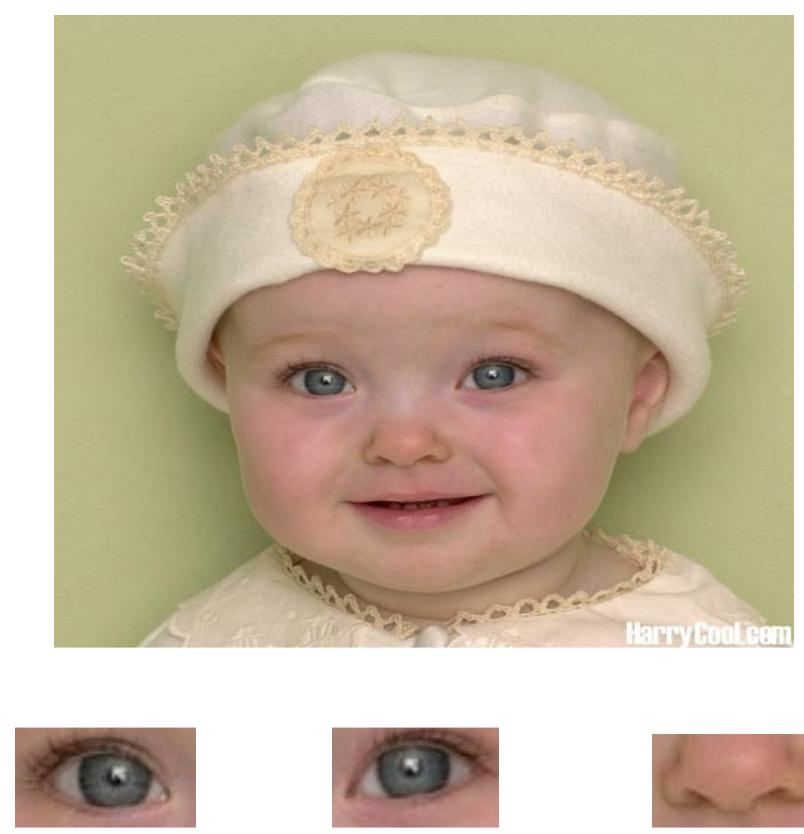

Template 1.1

Template 1.2

Fig. 2 Test image (1)
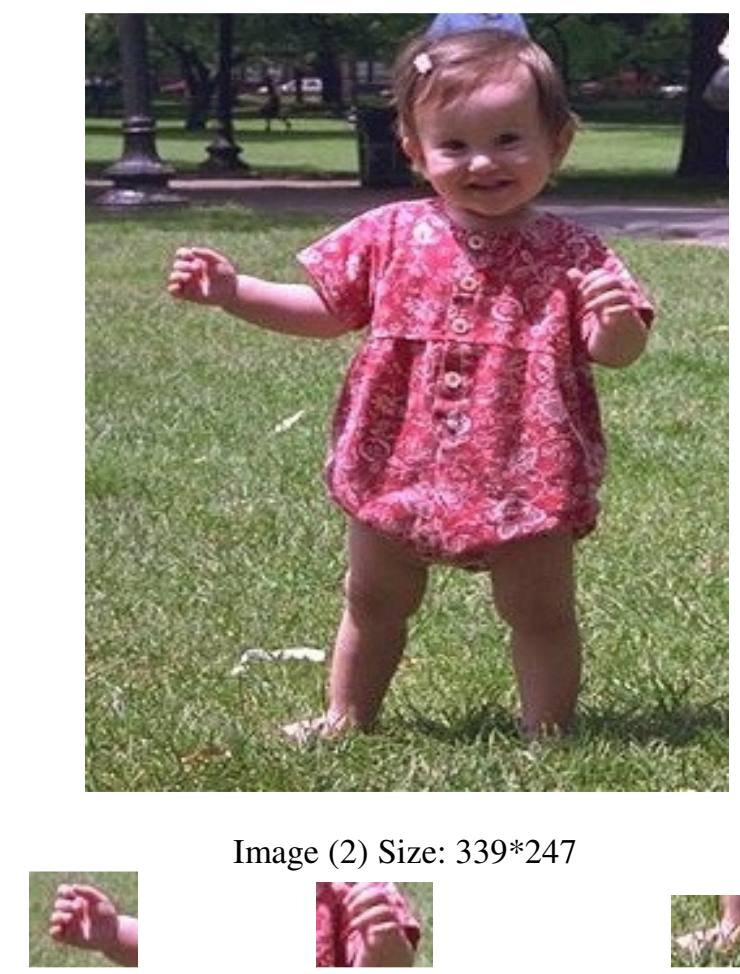

Template 2.1

Image (2) Size: 339*247

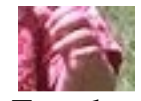

Template 2.2

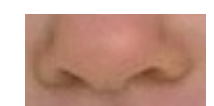

Template 1.2 
Signal \& Image Processing : An International Journal(SIPIJ) Vol.2, No.1, March 2011

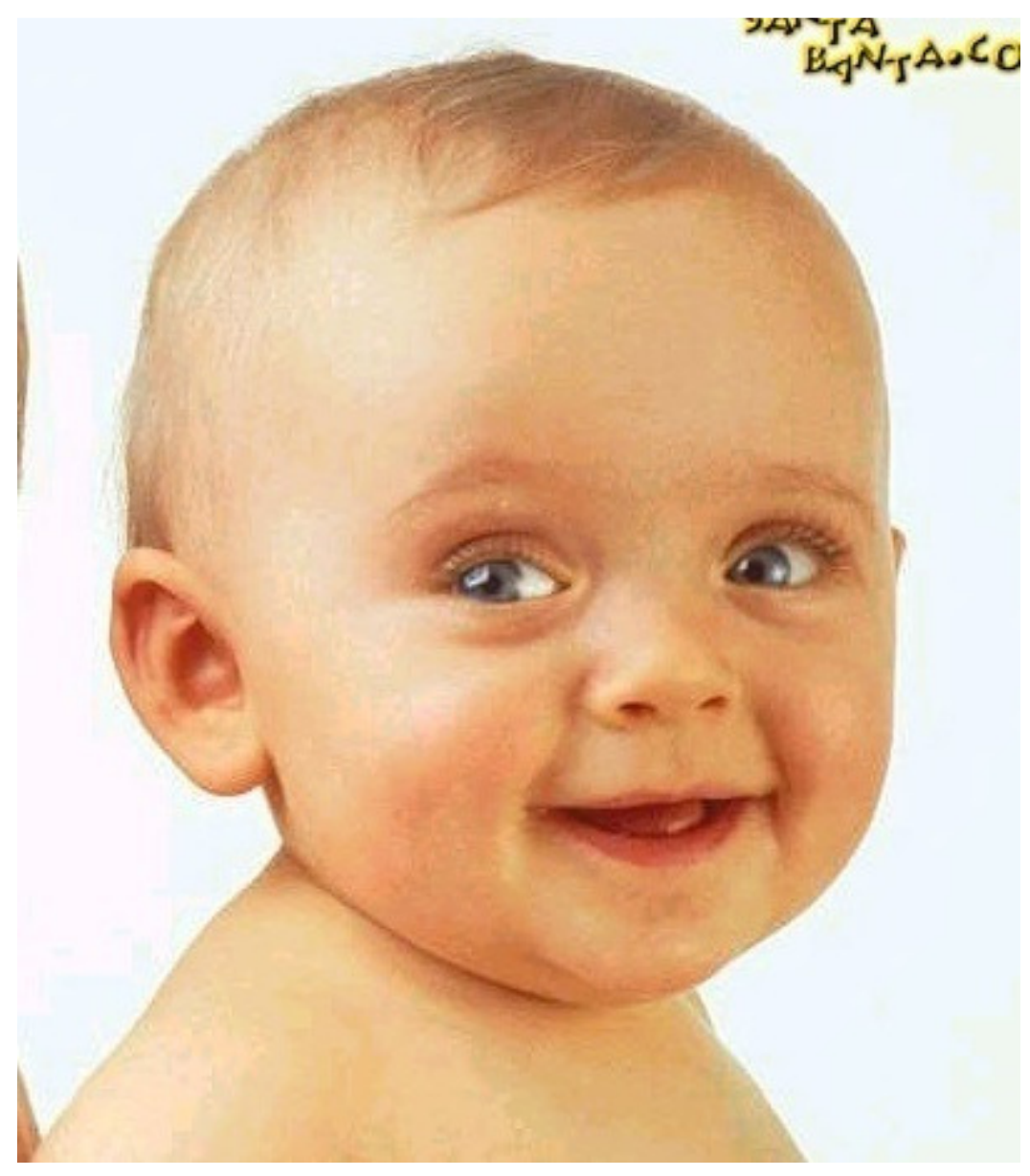

Image (3) Size: $459 * 402$

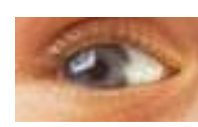

Template 3.1

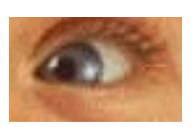

Template 3.2

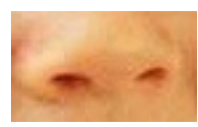

Template 3.3

Fig. 4 Test image (3) 
Signal \& Image Processing : An International Journal(SIPIJ) Vol.2, No.1, March 2011

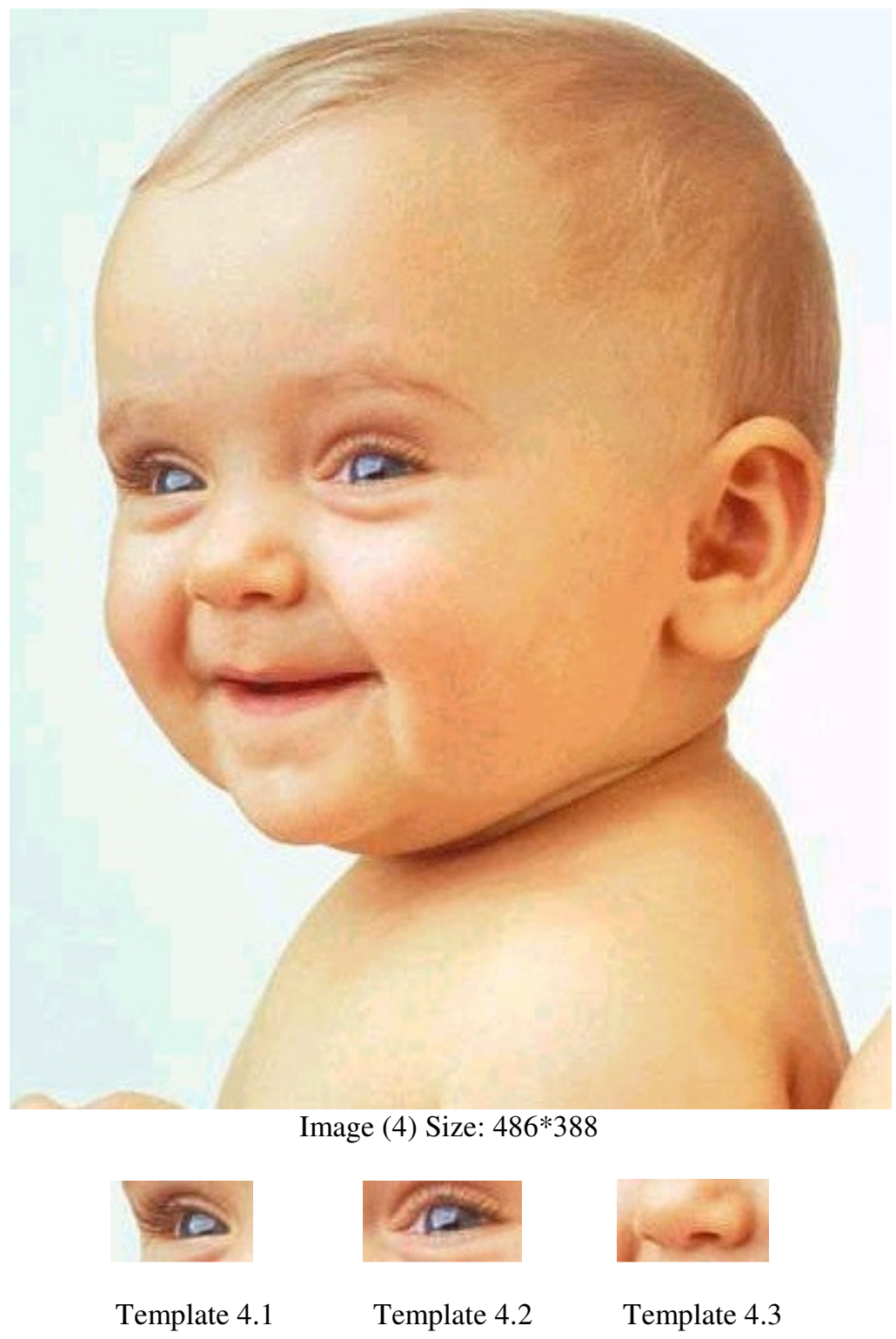

Fig. 5 Test image (4) 
Signal \& Image Processing : An International Journal(SIPIJ) Vol.2, No.1, March 2011



Image (5) Size: $427 * 410$



Template 5.1

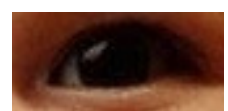

Template 5.2

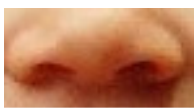

Template 5.3

Fig. 6 Test image (5)

The proposed technique was implemented in MATLAB and run under Pentium-4 (2.79 GHz) machine with 512 MB of RAM. Population size was fixed to 20. 
Signal \& Image Processing : An International Journal(SIPIJ) Vol.2, No.1, March 2011

\begin{tabular}{|c|c|c|c|c|}
\hline \multirow[t]{2}{*}{ Image No. } & \multirow[t]{2}{*}{ Template No. } & \multicolumn{2}{|c|}{ Modified PSO Based Algorithm } & \multirow{2}{*}{$\begin{array}{c}\begin{array}{c}\text { Old } \\
\text { Algorithm }\end{array} \\
\text { Time } \\
\text { Taken } \\
\text { (In sec.) }\end{array}$} \\
\hline & & No. of iteration & $\begin{array}{c}\text { Time Taken } \\
\text { (In sec.) }\end{array}$ & \\
\hline \multirow[t]{3}{*}{ Image (1) } & Template 1.1 & 200 & 4.50 & 71.38 \\
\hline & Template 1.2 & 200 & 4.60 & 276.90 \\
\hline & Template 1.3 & 200 & 4.50 & 142.59 \\
\hline \multirow[t]{3}{*}{ Image (2) } & Template 2.1 & 200 & 3.02 & 3.09 \\
\hline & Template 2.2 & 200 & 2.93 & 29.88 \\
\hline & Template 2.3 & 200 & 2.60 & 9.03 \\
\hline \multirow[t]{3}{*}{ Image (3) } & Template 3.1 & 200 & 4.78 & 61.38 \\
\hline & Template 3.2 & 200 & 4.71 & 210.14 \\
\hline & Template 3.2 & 200 & 5.26 & 164.50 \\
\hline \multirow[t]{3}{*}{ Image (4) } & Template 4.1 & 200 & 4.09 & 10.70 \\
\hline & Template 4.2 & 200 & 4.10 & 49.77 \\
\hline & Template 4.3 & 200 & 4.46 & 22.66 \\
\hline \multirow[t]{3}{*}{ Image (5) } & Template 5.1 & 200 & 5.10 & 29.17 \\
\hline & Template 5.2 & 200 & 5.31 & 147.85 \\
\hline & Template 5.3 & 200 & 5.00 & 77.90 \\
\hline
\end{tabular}

Table 3 Time consumed and No. of iterations by Modified PSO based algorithm \& Old algorithm 
Signal \& Image Processing : An International Journal(SIPIJ) Vol.2, No.1, March 2011

\section{CONCLUSION}

In the proposed work, we have successfully employed the PSO based methods to solve the object detection problem. The PSO based algorithm has superior features, including high-quality solution, stable convergence characteristic and good computation efficiency. The results show that the proposed method is capable of obtaining higher quality solution efficiency. It is clear from the results that the proposed PSO based method can avoid the shortcoming of Old template matching algorithm and can provide higher quality solution with better computation efficiency.

When the simple test images are tested on PSO based algorithm \& on PPO based algorithm for detecting the position of object then it is found that both of the algorithms are capable of detecting the position of object in image with very less time as compared to conventional template matching algorithm.In conventional template matching algorithm the time taken for detection of object is nearly $5 \mathrm{sec}$., while in the proposed algorithms the time consumed in the process of object detection is reduced by 10 times.PSO \& PPO based algorithms are tested on some real time images, $\&$ it is found that these algorithms are not performing well for finding the position of object in image. Concept of regenerating the population is used for the detection of object in such type of images \& on testing it is found that this algorithm is capable of detecting the position of the object with 10 times reduction in time as compared to conventional template matching algorithms.

\section{FUTURE SCOPE OF WORK}

Algorithms based on PSO proposed in this paper for object detection can be implemented in wide range of applications, e.g. to navigation, guidance, automatic surveillance, robot vision, and to the mapping sciences. Any automated system for three-dimensional point positioning can use proposed algorithms for object detection. For a computer vision system, the ability to cope with moving and changing objects, changing illumination, and changing viewpoints is essential to perform several tasks. Object detection is necessary for surveillance applications, for guidance of autonomous vehicles, for efficient video compression, for smart tracking of moving objects, for automatic target recognition (ATR) systems and for many other applications. In all of the above application the proposed algorithm can provide faster. 
Signal \& Image Processing : An International Journal(SIPIJ) Vol.2, No.1, March 2011

\section{REFERENCES}

[1] T. Peli, "An algorithm for recognition and localization of rotated and scaled objects", Proceedings of the IEEE 69 (1981) 483-485.

[2] Kun Peng, Liming Chen, Su Ruan, Georgy Kukharev, "A Robust and Efficient Algorithm for Eye Detection on Gray Intensity Face," Lecture Notes in Computer Science - Pattern Recognition and Image Analysis, pp. 302-308, 2005

[3] A. Goshtasby, S.H. Gage, and J.F. Bartholic, "A Two-Stage Cross Correlation Approach to Template Matching”, IEEE Trans. Pattern Analysis and Machine Intelligence, vol. 6, no. 3, pp. 374-378, 1984.

[4] FOERSTNER, W., "Quality assessment of object location and point transfer using digital image correlation techniques. International Archives of Photogrammetry and Remote Sensing" vol. XXV, A3a, Commission III, Rio de Janeiro, 1984.

[5] ACKERMANN, F. 1984. "Digital image correlation: Performance and potential application in photogrammetry". Photogrammetric Record 11 (64):429-439.

[6] J. P. Lewis, "Fast normalized cross-correlation", Canadian Image Processing and Pattern Recognition Society, Quebec City, Canada, May 15-19, 1995.

[7] J. Bala, K. DeJong, J. Huang, H. Vafaie, H. Wechsler, "Visual routine for eye detection using hybrid genetic architectures," International Conference on Pattern Recognition, vol. 3, pp. 606-610, 1996.

[8] Martin Berger, "The framework of least squares template matching.", 1998.

[9] Angeline, P. J., "Evolutionary optimization versus particle swarm optimization: philosophy and performance differences", The Seventh Annual Conf. on Evolutionary Programming, 1998.

[10] Shi, Y. and Eberhart, R. C., "Parameter selection in particle swarm optimisation" Evolutionary Programming VII: Proc. EP 98. New York, pp. 591-600, 1998.

[11] Shi, Y. and Eberhart, R. C., "Empirical study of particle swarm optimisation", Proceedings of the 1999 Congress on Evolutionary Computation. Piscataway, NJ, pp. 1945-1950, 1999.

[12] Kennedy, J., "Small worlds and mega-minds: effects of neighborhood topology on particle swarm performance", Proc. Congress on Evolutionary Computation 1999. Piscataway, NJ, pp. 1931-1938, 1999.

[13] Kennedy, J., Eberhart, R. C., and Shi, Y., "Swarm intelligence", Morgan Kaufmann Publishers, San Francisco. 2001.

[14] Kwan-Ho Lin, Kin-Man Lam and Wan-Chi Siu, "Locating the Eye in Human Face Images Using Fractal Dimensions," IEE Proceedings - Vision, Image and Signal Processing, vol. 148, no. 6, pp. 413-421, 2001.

[15] R.M. Dufour, E.L. Miller, N.P. Galatsanos, "Template matching based object recognition with unknown geometric parameters," IEEE Transactions on Image Processing, vol. 11, issue.12, pp. 1385 1396, 2002.

[16] Feng Zhao, Qingming Huang, Wen Gao, "Methods of image matching by normalized crosscorrelation".

[17] Mukesh Motwani, Rakhi Motwani, and Frederick C. Harris, Jr. "Eye Detection using wavelets and ANN," GSPx 2004, 2004.

[18] Z.-H. Zhou and X. Geng, "Projection functions for eye detection," Pattern Recognition, 37(5), pp. 1049-1056, 2004.

[19] Yacov Hel-Or, Hagit Hel-Or, "Real-Time Pattern Matching Using Projection Kernels", IEEE transactions on pattern analysis and machine intelligence, Vol. 27, No. 9, September, 2005.

[20] Serkan Kiranyaz, Turker Ince, Alper Yildirim and Moncef Gabbouj, "Fractional Particle Swarm Optimization in Multi-Dimensional Search Space," IEEE Transactions on Systems, Man, and Cybernetics:

Part B, vol. 40, no. 2, April 2010, pp.298-319. 
Signal \& Image Processing : An International Journal(SIPIJ) Vol.2, No.1, March 2011

\section{Authors}

Ankit Sharma received his M.E. degree from Thapar University Patiala in 2010. Currently he is working as Assistant professor in Electronics \& Instrumentation Engineering department at SVITS, Indore, India.His areas of intrest include Digital image Processing, embedded system and optimization.

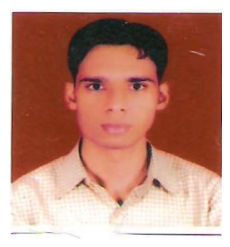

Nirbhow Jap singh is working as Assistant professor in Electrical and Instrumentation engineering department at Thapar university, patiala, Punjab. He has teaching experience of 8 yrs. He is currently perusing his $\mathrm{PhD}$. in the field of optimization and its applications at Thapar university, patiala. His areas of interest are soft computing, embedded systems, industrial automation and mechatronics. 